Journal Of Al-Azhar University Engineering Sector

Vol. 14, No. 50, January, 2019, 109-120

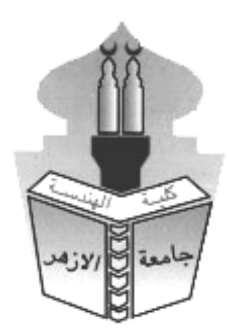

\title{
SOLUTION OF GENERAL FORM OF FUZZY SECOND ORDER BOUNDARY VALUE PROBLEM
}

\author{
M. shokry and B. Kamal \\ Departement of ph. and Math. -Faculty of Eng.-Tanta university
}

\begin{abstract}
In this paper; we obtained a new method for solving the homogenous second order linear differential equation with boundary condition in fuzzy environment under strong generalized Hukuhara differentiability and illustrated this by several examples.

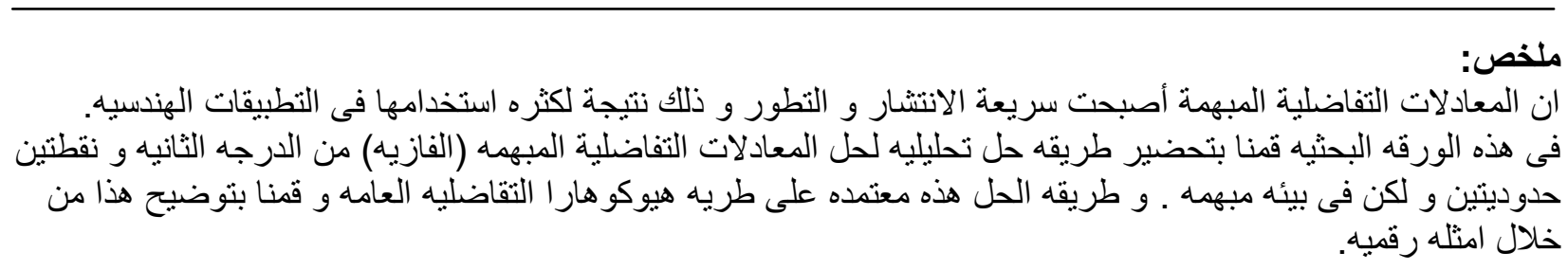

\section{KEYWORDS: Fuzzy Set Theory, Fuzzy Analysis, Fuzzy Differential Equations.}

\section{INTRODUCTION}

Fuzzy differential equation (FDE) has been rapidly developing in recent years, and has attracted many researchers. The use of FDE is a smart way to model dynamic systems under uncertain information [25].The nation of fuzzy derivative was first induced by (Zadeh and Chang) [9], it was followed up by (Dubois and prade) [10], also other process has been discussed by (Puri and Ralescu and Goetschel and Voxman) [20,12]. The concept of differential equation in fuzzy environment concepts was formulated by (Kaleva) [17], using of Hukuhara or generalized derivatives the solution turns fuzzier as time goes by [11]. But (Bede) found that a large class of BVPs has no solution if hukuhara derivative is used [3], so to overcome this, the concept of generalized derivative was developed $[2,7]$. (Khastan and Nieto) found solutions of a large class of BVPs using the generalized derivative [18].

(Stefanini and Bede) by the concept of generalization of hukuhara difference for compact convex set and introduced generalized hukuhara differentiability for fuzzy valued function, the demonstrated that $[2,23]$. Recently, (Gasilov) solve the fuzzy initial value problem by a new technique (linear transformation) [13] and (Barros) solve fuzzy differential equation by fuzzification of the derivative operator [6].

Due to the wide applications of the second order fuzzy differential equation, it is considered as the most important between all fuzzy differential equations, So, Many researchers have worked on the second FDE, (Wang and Gue) [28] solve second order by adomian, (Gasilov) [13, 14] solve by linear transformation, (Ahmadi) apply fuzzy Laplace transform [20], (jamoshidi and Avazpour) found way by shooting method [16], while (Rabiei) solved by improved runge kutta [22], Finally (Mondal and ray) solved in fuzzy environment analytically [26]. 


\section{BASIC CONCEPTS}

In this section, we will illustrate the fundamental concepts and facts related to fuzzy differential equations. According to Zadeh [25], a fuzzy set is a generalization of a classical set that allows the membership function to take any value in the unit interval $[0,1]$.

Definition 2.1 1 1-4] Let $U$ a nonempty universe and fuzzy set $(A)$ in $U$ is a function $A: U \rightarrow[0,1]$,Where $\mu(x)$ is the degree of membership of $x$ in $A$, when $\mu(x)$ goes closer to 1 , the $X$ is more considered to belong to $A$, but when it goes closer to 0 , the $X$ is less considered to belong to $A . A=\{(x, \mu(x)), x \in X\}$

Definition 2.2 [1-4]Let $A$ be a fuzzy set in $U$, the support of $A$ is the crisp set in all elements in $U$ with non-zero membership in $\operatorname{S} \operatorname{Supp}(A)=\{x \in A \mid A(x)>0\}$

Definition2.3[1-4] Let $A$ be a fuzzy set in $U$, the core of $A$ is the crisp set in all elements in $U$ with membership in $A$ equals 1.Core $(A)=\{x \in A \mid A(x)=1\}$

Definition 2.4[1-4] Let $A$ be a fuzzy set in $R$. $A$ Is called a fuzzy interval if:

(i) $A$ is normal: there exists $x_{0} \in R$ then $A\left(x_{0}\right)=1$

(ii) $A$ is Convex: for all $x, y \in R$ and $0 \leq \lambda \leq 1$, it holds that

$$
A(\lambda x+(1-\lambda) y) \geq \min (A(x), A(y)) \text {; }
$$

(iii) $A$ is upper semi-continuous

$$
A\left(x_{0}\right) \geq \lim _{x \rightarrow x_{0}^{ \pm}} A(x) ;
$$

(iv) $[A]^{0}=\overline{\operatorname{supp}(A)}$ is compact subset of $\mathrm{R}$

Definition 2.5:[12,23]Let $A$ be a fuzzy set then, $\alpha-$ cut of AIs the crisp set of $[A]^{\alpha}$ that contains all elements with membership greater than or equal $\alpha$. Where; $\alpha \in] 0,1][A]^{\alpha}=\{x \in R \mid A(x) \geq \alpha\}$. $[A]^{\alpha}=\left[a_{1}^{\alpha}, a_{2}^{\alpha}\right]$. Where $a_{1}^{\alpha}$ is lower and $a_{2}^{\alpha}$ is upper.

Definition 2.6 [3,4 ]The fuzzy number is an extension of a regular number but, it does not refer to a single value. It refers to a connected set of possible values, where each possible value has its own membership from 0 to 1 . Thus fuzzy number is a convex and normal fuzzy set.

\section{Definition 2.7: generalized trapezoidal fuzzy number( $G \operatorname{Tr} F N)$ [17]}

$\bar{A}_{G T F N}$ is a subset of IFN in $R$ with following membership:

$\bar{A}_{\text {GTFN }}=\left(a_{1}, a_{2}, a_{3}, a_{4}, \omega\right)$;

$\mu(x)=\left\{\begin{array}{cc}\omega \frac{x-a_{1}}{a_{2}-a_{1}} & \text { if } a_{1} \leq x \leq a_{2} \\ \omega & \text { if } a_{2} \leq x \leq a_{3} \\ \omega \frac{a_{4}-x}{a_{4}-a_{3}} & \text { if } a_{3} \leq x \leq a_{4} \\ 0 & \text { otherwise }\end{array} ;\right.$

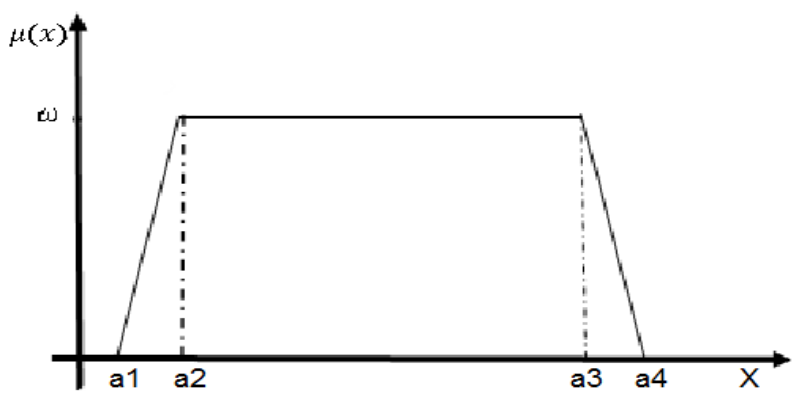

Fig. 1: $G \operatorname{Tr} F N$ representation 
At $\omega=1$, we can get trapezoidal fuzzy number.

Definition 2.8 [3,17]: Generalized triangle fuzzy number $G \operatorname{Tr} F N$

We can say that this number is a special case of generalized trapezoidal fuzzy number when the core becomes point not interval.

$$
\begin{aligned}
& \bar{A}_{G T F N}=\left(a_{1}, a_{2}, a_{3}, \omega\right) ; \\
& \mu(x)=\left\{\begin{array}{cc}
\omega \frac{x-a_{1}}{a_{2}-a_{1}} & \text { if } a_{1} \leq x \leq a_{2} \\
\omega & \text { if } a_{2}=x \\
\omega \frac{a_{3}-x}{a_{3}-a_{2}} & \text { if } a_{2} \leq x \leq a_{3} \\
0
\end{array}\right.
\end{aligned}
$$

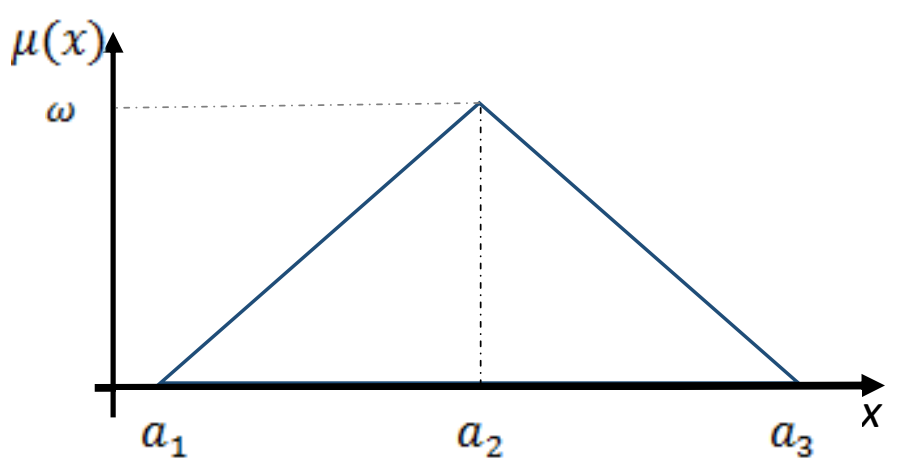

Fig. 2: triangle fuzzy number

At $\omega=1$, we can get triangle fuzzy number.

\section{Definition 2.9: [11], distance between two fuzzy intervals}

Let $A$ and $B$ are two fuzzy intervals then,Hausedorff distance [11] between $[A]^{\alpha}$ and $[b]^{\alpha}$ is; $d_{H}\left([A]^{\alpha},[B]^{\alpha}\right)=\max \left\{\left|a_{1}^{\alpha}-b_{1}^{\alpha}\right|,\left|a_{2}^{\alpha}-b_{2}^{\alpha}\right|\right\}$ By using Hausedorff distance, it is easily to find the distance between two fuzzy intervals which can be written as following $D(A, B)=\sup _{\alpha \in[0,1]} d_{H}\left([A]^{\alpha},[B]^{\alpha}\right)$.

Definition 2.10: [10], Let $x, y$ and $z$ are fuzzy numbers, and there exists $x=y+z$ Then, Hukuhara difference is $z=x \ominus y=\left[z_{1}^{\alpha}, z_{2}^{\alpha}\right]=\left[x_{1}^{\alpha}, x_{2}^{\alpha}\right] \ominus\left[y_{1}^{\alpha}, y_{2}^{\alpha}\right]=\quad\left[x_{1}^{\alpha}-y_{1}^{\alpha}, x_{2}^{\alpha}-y_{2}^{\alpha}\right]$ ,Where $x \ominus y \neq x+(-y)$.

Then, Generalized Hukuhara difference is $z=x \ominus_{g h} y=\left[z_{1}^{\alpha}, z_{2}^{\alpha}\right]$ then; $z_{1}^{\alpha}=\min \left[\left(x_{1}^{\alpha}-y_{1}^{\alpha}\right),\left(x_{2}^{\alpha}-y_{2}^{\alpha}\right)\right], z_{2}^{\alpha}=\max \left[\left(x_{1}^{\alpha}-y_{1}^{\alpha}\right),\left(x_{2}^{\alpha}-y_{2}^{\alpha}\right)\right]$.

Definition 2.11: [4-7,23], The generalized hukuhara first derivative of a fuzzy parametric function is defined as; $f^{\prime}\left(t_{0}\right)=\lim _{h \rightarrow 0} \frac{f\left(t_{0}+h\right) \ominus_{g h} f\left(t_{0}\right)}{h}$,From the definition, we have two classes:

(i)-differentiable at $t_{0} \quad\left[f^{\prime}\left(t_{0}\right)\right]_{\alpha}=\left[f_{1}^{\prime}\left(t_{0}, \alpha\right), f_{2}^{\prime}\left(t_{0}, \alpha\right)\right]$

(ii)-differentiable at $t_{0} \quad\left[f^{\prime \prime}\left(t_{0}\right)\right]_{\alpha}=\left[f_{2}^{\prime \prime}\left(t_{0}, \alpha\right), f_{1}^{\prime}\left(t_{0}, \alpha\right)\right]$ Where, $f_{1}$ is the lower and $f_{2}$ is the upper.

Definition 2.12 [23, 24], The generalized hukuhara second derivative of fuzzy function is defined as; $f^{\prime \prime}\left(t_{0}\right)=\lim _{h \rightarrow 0} \frac{f^{\circ}\left(t_{0}+h\right) \ominus_{g h} f^{\prime \prime}\left(t_{0}\right)}{h}$

According to the Definition 12, we have the following classes: $f^{\prime}\left(t_{0}\right)$ is $(i)$--differentiable if: 
SOLUTION OF GENERAL FORM OF FUZZY SECOND ORDER BOUNDARY VALUE PROBLEM

$$
f^{\mathrm{m}}\left(t_{0}\right)=\left\{\begin{array}{c}
{\left[f_{1}^{\mathrm{m}}\left(t_{0}, \alpha\right), f_{2}^{\mathrm{m}}\left(t_{0}, \alpha\right)\right] \text { if } f \text { is }(i) \text {-differentiable }} \\
\text { class }(1,1) \\
{\left[f_{2}^{\mathrm{m}}\left(t_{0}, \alpha\right), f_{1}^{\mathrm{m}}\left(t_{0}, \alpha\right)\right] \text { if } f \text { is }(\text { ii })-\text { differentiable }} \\
\text { class }(2,2)
\end{array}\right\}
$$

$f^{\prime \prime}\left(t_{0}\right)$ Is (ii)-differentiable if:

$f^{\prime \prime}\left(t_{0}\right)=\left\{\begin{array}{c}{\left[f_{2}^{\prime \prime}\left(t_{0}, \alpha\right), f_{1}^{\mathrm{m}}\left(t_{0}, \alpha\right)\right] \text { if } f \text { is }(i)-\text { differentiable }} \\ \text { class }(1,2) \\ {\left[f_{1}^{\mathrm{m}}\left(t_{0}, \alpha\right), f_{2}^{\mathrm{m}}\left(t_{0}, \alpha\right)\right] \text { if } f \text { is }(\text { ii })-\text { differentiable }} \\ \text { class }(2,1)\end{array}\right\}$

Definition 2.13: [26] $\operatorname{Let}\left[x_{1}(t, \alpha), x_{2}(t, \alpha)\right]$ be solution of any fuzzy differential is called a strong solution, if $\frac{d x_{1}(t, \alpha)}{d \alpha}>0, \frac{d x_{2}(t, \alpha)}{d \alpha}<0 \forall \alpha \in[0, \omega], x_{1}(t) \leq x_{2}(t)$ Otherwise it is called a weak solution.

\section{Fuzzy boundary value problems (FBVP)}

In this section, the study concerns with the fuzzy effect and using the generalized Hukuhara differentiability on the following FBVP [24]:

$\tilde{y}^{I I}(x)=p \tilde{y}^{\prime}(x)+q \tilde{y}(x)$

According to the following fuzzy boundary conditions

$$
\tilde{y}\left(x_{0}\right)=\tilde{a}, \quad \tilde{y}\left(x_{b}\right)=\tilde{b},
$$

Where $p$ and $q$ are constants, åand $\tilde{b}$ are two generalized trapezoidal fuzzy number represented as:

$\tilde{a}=\left(a_{1}, a_{2}, a_{3}, a_{4} ; \omega\right)$ And $\widetilde{b}=\left(b_{1}, b_{2}, b_{3}, b_{4} ; \omega\right)$.

The lower and upper values of $\tilde{a}$ and $\tilde{b}$ are given according to Table 1:

\begin{tabular}{|l|l|l|}
\hline & Lower values & Upper values \\
\hline$\tilde{a}=[a, \bar{a}]_{\alpha}$ & $\underline{a}=a_{1}+\frac{\alpha\left(a_{2}-a_{1}\right)}{\omega}$ & $\bar{a}=a_{4}-\frac{\alpha\left(a_{4}-a_{3}\right)}{\omega}$ \\
\hline$\tilde{b}=[\underline{b}, \bar{b}]_{\alpha}$ & $\underline{b}=b_{1}+\frac{\alpha\left(b_{2}-b_{1}\right)}{\omega}$ & $\bar{b}=b_{4}-\frac{\alpha\left(b_{4}-b_{3}\right)}{\omega}$ \\
\hline
\end{tabular}

table 1: the lower and upper values

\section{SOLUTION OF FUZZY BOUNDARY VALUE PROBLEMS}

\section{Class $(1,1)$}

The FBVP(1) can be written as:

$$
\begin{array}{ll}
\overline{y^{\prime \prime}}(x, \alpha)=p \cdot \bar{y}^{\prime}(x, \alpha)+q \cdot \bar{y}(x, \alpha), & \bar{y}\left(x_{0}, \alpha\right)=\bar{a}, \quad \bar{y}\left(x_{b}, \alpha\right)=\bar{b}, \\
\text { And } & \underline{y}\left(x_{0}, \alpha\right)=\underline{a}, \underline{y}\left(x_{b}, \alpha\right)=\underline{b} . \\
\underline{y^{\prime \prime}}(x, \alpha)=p \cdot \underline{y^{\prime}}(x, \alpha)+q \cdot \underline{y}(x, \alpha), &
\end{array}
$$


In order to get the solution of $(3,4)$, we write these equations in the following system:

$$
\left[\begin{array}{l}
r \\
u \\
z \\
W
\end{array}\right]^{r}=\left[\begin{array}{llll}
0 & 1 & 0 & 0 \\
q & p & 0 & 0 \\
0 & 0 & 0 & 1 \\
0 & 0 & q & p
\end{array}\right]\left[\begin{array}{c}
r \\
u \\
z \\
W
\end{array}\right]
$$

Where $\underline{y}=r$ and $\bar{y}=z$.

So the lower and upper solutions are given by:

$\underline{y}(x, \alpha)=c_{2} e^{\frac{d_{1} x}{2}}+c_{4} e^{\frac{d_{3} x}{2}}$,

(6)

$\bar{y}(x, \alpha)=c_{1} e^{\frac{d_{1} x}{2}}+c_{3} e^{\frac{d_{3} x}{2}}$,

(7)

Where, $d_{1}, d_{2}, d_{3}$ and $d_{4}$ are constants given by:

$$
\begin{array}{ll}
d_{1}=p+\sqrt{p^{2}+4 q}, & d_{2}=p+\sqrt{p^{2}-4 q}, \\
d_{3}=p-\sqrt{p^{2}+4 q}, & d_{4}=p-\sqrt{p^{2}-4 q}
\end{array}
$$

Andthe constants $c_{1}, c_{2}, c_{3}$ and $c_{4}$ can be obtained by applying the fuzzy boundary condition given by Eq. $(3,4)$.

By using the value of $c, \underline{a}, \bar{a}, \underline{b}$ and $\bar{b}$ then:

$$
\begin{aligned}
& \underline{y}(x, \alpha)=\frac{\underline{a}\left(e^{\frac{d_{1} x_{b}+d_{3} x}{2}}-e^{\frac{d_{3} x_{b}+d_{1} x}{2}}\right)+\underline{b}\left(e^{\frac{d_{3} x_{0}+d_{1} x}{2}}-e^{\frac{d_{1} x_{0}+d_{3} x}{2}}\right)}{\left(e^{\frac{d_{1} x_{b}+d_{3} x_{0}}{2}}-e^{\frac{d_{3} x_{b}+d_{1} x_{0}}{2}}\right)} \\
& \bar{y}(x, \alpha)=\frac{\bar{a}\left(e^{\frac{d_{1} x_{b}+d_{3} x}{2}}-e^{\frac{d_{3} x_{b}+d_{1} x}{2}}\right)+\bar{b}\left(e^{\frac{d_{3} x_{0}+d_{1} x}{2}}-e^{\frac{d_{1} x_{0}+d_{3} x}{2}}\right)}{\left(e^{\frac{d_{1} x_{b}+d_{3} x_{0}}{2}}-e^{\frac{d_{3} x_{b}+d_{1} x_{0}}{2}}\right)}
\end{aligned}
$$

Where, $\underline{a}, \bar{a}, \underline{b}$ and $\bar{b}$ values from table 1 .

Class $(1,2)$

$$
\begin{aligned}
& \underline{y^{\prime \prime}}(x, \alpha)=p \cdot \bar{y}^{\prime}(x, \alpha)+q \cdot \bar{y}(x, \alpha), \bar{y}\left(x_{0}, \alpha\right)=\bar{a}, \bar{y}\left(x_{b}, \alpha\right)=\bar{b}, \\
& \overline{y^{\prime \prime}}(x, \alpha)=p \cdot \underline{y^{\prime}}(x, \alpha)+q \cdot \underline{y}(x, \alpha), \underline{y}\left(x_{0}, \alpha\right)=\underline{a}, \underline{y}\left(x_{b}, \alpha\right)=\underline{b},
\end{aligned}
$$

The general solution:

$$
\begin{aligned}
& \underline{y}(x, \alpha)=c_{1} e^{\frac{-x}{2} d_{2}}+c_{2} e^{\frac{-x}{2} d_{4}}+c_{3} e^{\frac{x}{2} d_{3}}+c_{4} e^{\frac{x}{2} d_{1}}, \\
& \bar{y}(x, \alpha)=c_{1} e^{\frac{-x}{2} d_{2}}\left(1-d_{4}(p / q)\right)+c_{2} e^{\frac{-x}{2} d_{4}}\left(1-d_{2}(p / q)\right)+c_{3} e^{\frac{x}{2} d_{n}}+c_{4} e^{\frac{x}{2} d_{1}}
\end{aligned}
$$

\section{Class $(2,1)$}

$\overline{y^{\prime \prime}}(x, \alpha)=p \cdot \bar{y}^{\prime}(x, \alpha)+q \cdot \underline{y}(x, \alpha), \bar{y}\left(x_{0}, \alpha\right)=\bar{a}, \bar{y}\left(x_{b}, \alpha\right)=\bar{b}$, 
$\underline{y^{\prime \prime}}(x, \alpha)=p \cdot \underline{y^{\prime}}(x, \alpha)+q \cdot \bar{y}(x, \alpha), \underline{y}\left(x_{0}, \alpha\right)=\underline{a}, \underline{y}\left(x_{b}, \alpha\right)=\underline{b}$,

The general solution:

$\underline{y}(x, \alpha)=c_{1} e^{\frac{x}{2} d_{2}}+c_{2} e^{\frac{x}{2} d_{1}}+c_{3} e^{\frac{x}{2} d_{4}}+c_{4} e^{\frac{x}{2} d_{3}}$

$\bar{y}(x, \alpha)=-c_{1} e^{\frac{x}{2} d_{2}}+c_{2} e^{\frac{x}{2} d_{1}}-c_{3} e^{\frac{x}{2} d_{4}}+c_{4} e^{\frac{x}{2} d_{3}}$

Class $(2,2)$

$\overline{y^{\prime \prime}}(x, \alpha)=p \cdot y^{\prime}(x, \alpha)+q \cdot \bar{y}(x, \alpha), \bar{y}\left(x_{0}, \alpha\right)=\bar{a}, \bar{y}\left(x_{b}, \alpha\right)=\bar{b}$,

$\underline{y^{\prime \prime}}(x, \alpha)=p \cdot \bar{y}^{\prime \prime}(x, \alpha)+q \cdot \underline{y}(x, \alpha), \underline{y}\left(x_{0}, \alpha\right)=\underline{a}, \underline{y}\left(x_{b}, \alpha\right)=\underline{b}$,

The general solution:

$$
\begin{aligned}
& \underline{y}(x, \alpha)=c_{1} e^{\frac{-x}{2} d_{3}}+c_{2} e^{\frac{x}{2} d_{3}}+c_{3} e^{\frac{-x}{2} d_{1}}+c_{4} e^{\frac{x}{2} d_{1},} \\
& \bar{y}(x, \alpha)=-c_{1} e^{\frac{-x}{2} d_{3}}+c_{2} e^{\frac{x}{2} d_{3}}-c_{3} e^{\frac{-x}{2} d_{1}}+c_{4} e^{\frac{x}{2} d_{1}},
\end{aligned}
$$

\section{Example 4.1.}

Consider the following FBVP

$$
\widetilde{y^{\prime \prime}}(t)=5 \widetilde{y}^{\prime}(t)+4 \widetilde{y}(t), \widetilde{y}(0)=(0.8,1,1.1,1.3 ; 0.7), \widetilde{y}(1)=(2.6,2.8,3,3.4 ; 0.7) \text {. }
$$

\section{Class $(1,1)$}

The general solution is given by

$$
\begin{aligned}
& \underline{y}(t, \alpha)=(0.48 \alpha+7.3) 10^{-3} e^{\frac{(5+\sqrt{41}) t}{2}}+(0.285 \alpha+0.79) e^{\frac{(5-\sqrt{41}) t}{2}}, \\
& \bar{y}(t, \alpha)=(9.2-1.4 \alpha) 10^{-3} e^{\frac{(5+\sqrt{41}) t}{2}}+(1.29-0.284 \alpha) e^{\frac{(5-\sqrt{41}) t}{2}} .
\end{aligned}
$$

Also, for different values of $\alpha$, we plotted the lower and upper solutions in Fig. 3, and we listed the lower and upper solutions for $t=0.5$ in Table 2 .

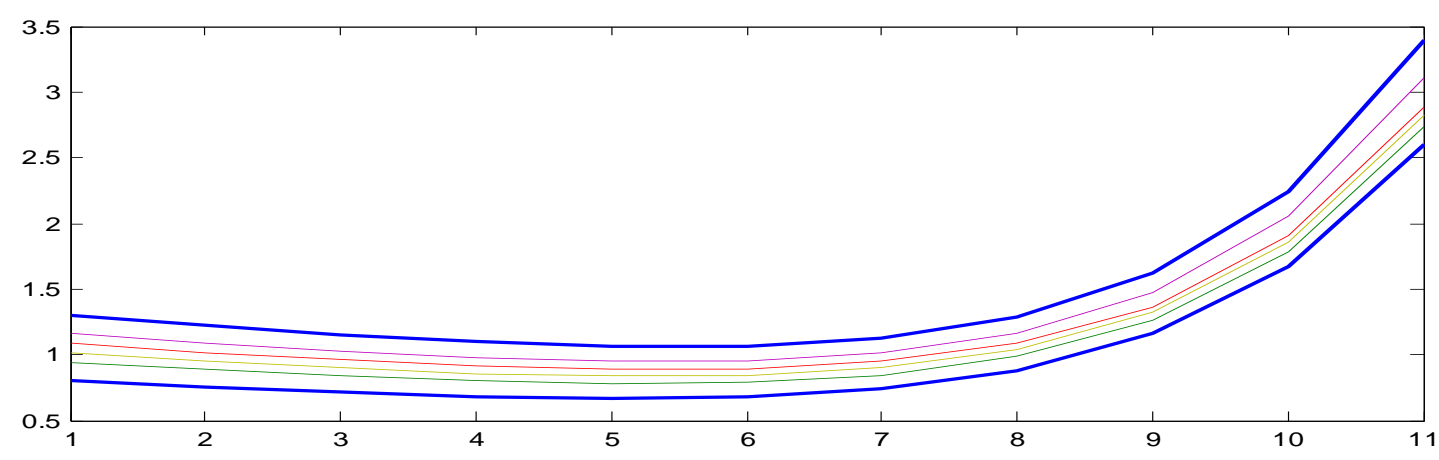

Fig. 3:The lower and upper solution class $(1,1)$ of Example 1for $\alpha=0,0.5,0.7$

\section{Class $(1,2)$}

The general solution is given by 
$\underline{y}(t, \alpha)=$

$$
\begin{aligned}
& (0.7-0.74 \alpha) e^{-4 t}+(0.242 \alpha-0.226) e^{-t}+(0.78 \alpha+0.312) e^{\frac{(5-\sqrt{41}) t}{2}}+ \\
& (0.008-0.0059 \alpha) e^{\frac{(5+\sqrt{41}) t}{2}}
\end{aligned}
$$$$
\bar{y}(t, \alpha)=(0.7-0.74 \alpha) e^{-4 t}(-(3 / 2))+(0.242 \alpha-0.226) e^{-t}(-9)+
$$$$
(0.78 \alpha+0.312) e^{\frac{(5-\sqrt[3]{41}) t}{2}}+(0.008-0.0059 \alpha) e^{\frac{(5+\sqrt[3]{41}) t}{2}}
$$

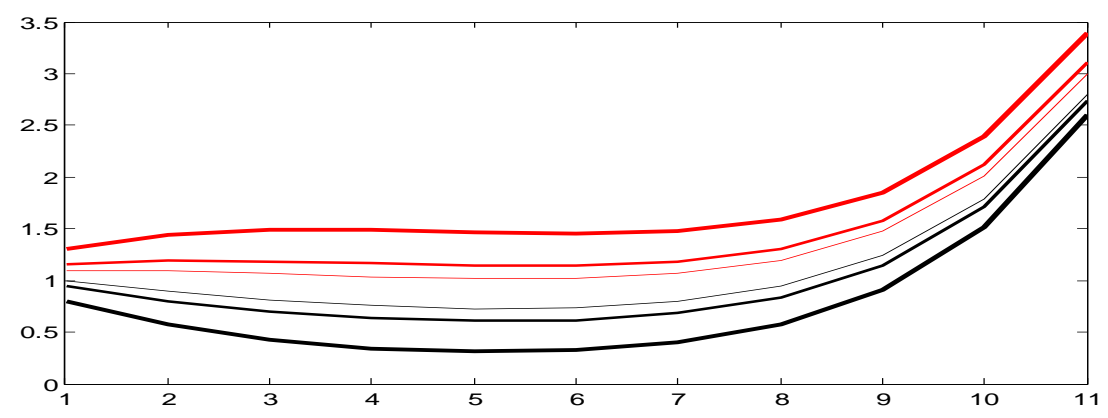

Fig. 4:The lower and upper solution class $(1,2)$ of Example 1for $\alpha=0,0.5,0.7$

\section{Class $(2,1)$}

The general solution is given by

$$
\begin{gathered}
\underline{y}(t, \alpha)=(0.0054-0.0067 \alpha) e^{4 t}+(0.0083-0.00047 \alpha) e^{\frac{(5+\sqrt[3]{41}) t}{2}} \\
+(0.29 \alpha-0.255) e^{t}+(0.00047 \alpha+1.04) e^{\frac{(5-\sqrt[4]{41}) t}{2}}, \\
\bar{y}(t, \alpha)=-(0.0054-0.0067 \alpha) e^{4 t}+(0.0083-0.00047 \alpha) e^{\frac{(5+\sqrt[3]{41}) t}{2}} \\
-(0.29 \alpha-0.255) e^{t}+(0.00047 \alpha+1.04) e^{\frac{(5-\sqrt[3]{41}) t}{2}}
\end{gathered}
$$

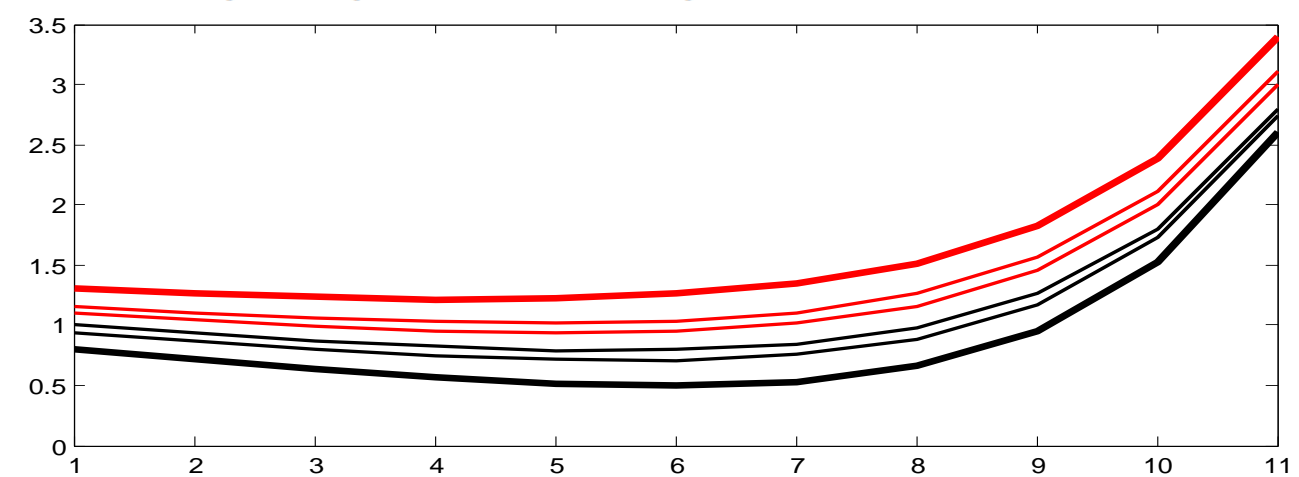

Fig. 5:The lower and upper solution class $(2,1)$ of Example 1for $\alpha=0,0.5,0.7$

\section{Class $(2,2)$}

The general solution is given by 
SOLUTION OF GENERAL FORM OF FUZZY SECOND ORDER BOUNDARY VALUE PROBLEM
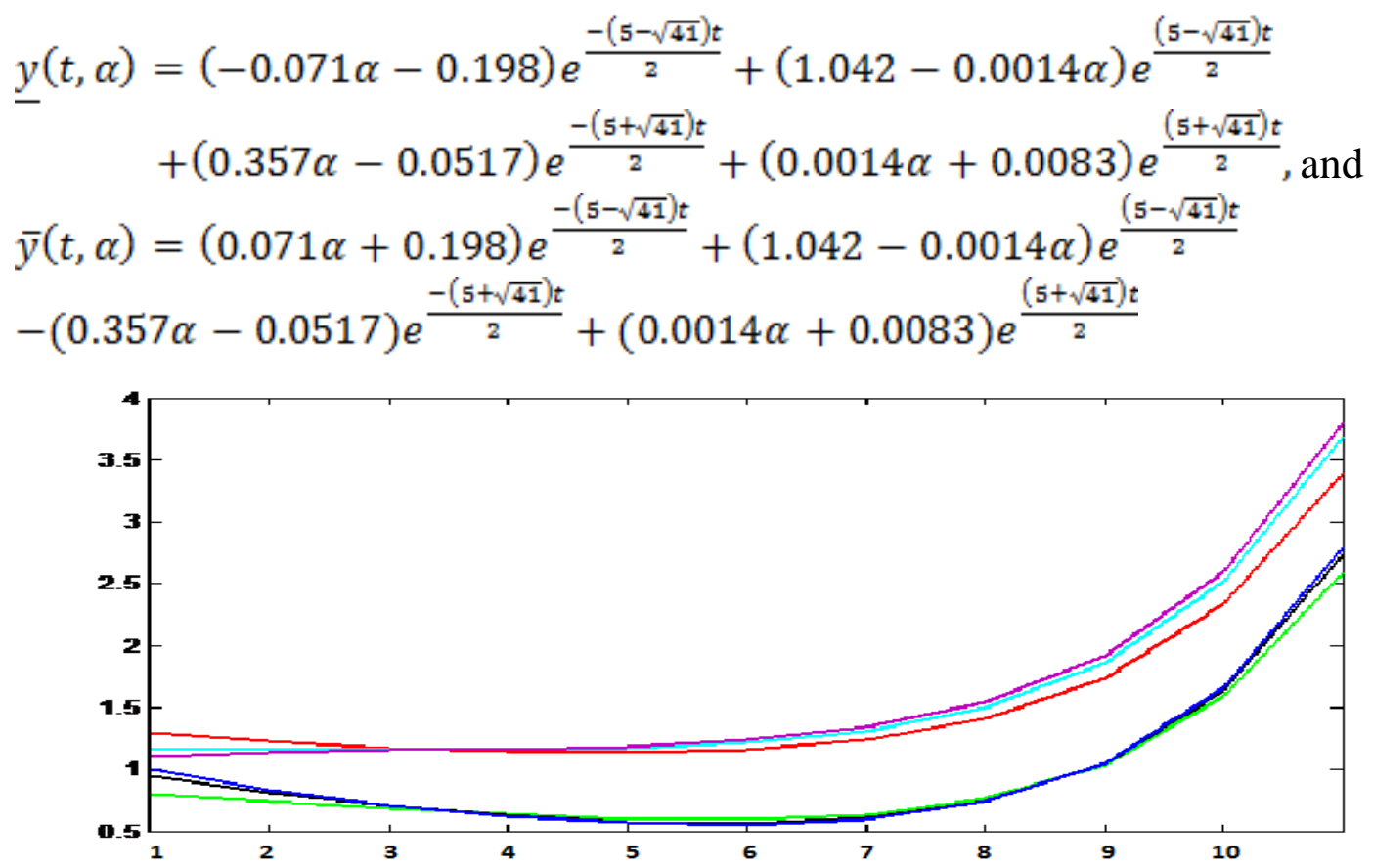

Fig. 6:The lower and upper solution class (2,2) of Example 1for $\alpha=0,0.5,0.7$

From Fig. 6, we notice the intersection between the solution and this indicates that this class represents a weak solution which means that the inner band may become outer band at different $\mathrm{t}$ so, we can check the variation with $\alpha$ in Table 2.

Table 2: The lower and upper solutions of Example 1 for $t=0.5$

\begin{tabular}{|l|l|l|l|l|l|l|l|l|}
\hline & \multicolumn{2}{|l|}{ Class $(1,1)$} & \multicolumn{2}{l|}{ Class $(1,2)$} & \multicolumn{2}{l|}{ Class $(2,1)$} & \multicolumn{2}{l|}{ Class $(2,2)$} \\
\hline$\alpha$ & $\underline{y}(t, \alpha)$ & $\bar{y}(t, \alpha)$ & $\underline{y}(t, \alpha)$ & $\bar{y}(t, \alpha)$ & $\underline{y}(t, \alpha)$ & $\bar{y}(t, \alpha)$ & $\underline{y}(t, \alpha)$ & $\bar{y}(t, \alpha)$ \\
\hline 0 & 0.6857 & 1.0684 & 0.3239 & 1.4576 & 0.4958 & 1.2583 & 0.5925 & 1.1616 \\
\hline 0.1 & 0.7066 & 1.0459 & 0.3827 & 1.3946 & 0.5383 & 1.2142 & 0.5868 & 1.1720 \\
\hline 0.2 & 0.7275 & 1.0234 & 0.4416 & 1.3316 & 0.5807 & 1.1702 & 0.5811 & 1.1825 \\
\hline 0.3 & 0.7484 & 1.0009 & 0.5005 & 1.2686 & 0.6232 & 1.2610 & 0.5754 & 1.1930 \\
\hline 0.4 & 0.7694 & 0.9784 & 0.5594 & 1.2056 & 0.6656 & 1.0821 & 0.5697 & 1.2034 \\
\hline 0.5 & 0.7903 & 0.9559 & 0.6182 & 1.1426 & 0.7081 & 1.0381 & 0.5640 & 1.2139 \\
\hline 0.6 & 0.8112 & 0.9334 & 0.6771 & 1.0796 & 0.7506 & 0.9940 & 0.5583 & 1.2243 \\
\hline 0.7 & 0.8321 & 0.9109 & 0.7360 & 1.0166 & 0.7930 & 0.9500 & 0.5526 & 1.2348 \\
\hline
\end{tabular}

From Table 2, we conclude that $\underline{y}(t, \alpha)$ is increasing and $\bar{y}(t, \alpha)$ is decreasing Then, the solution is a strong solution for class $(1,1),(1,2)$ and $(2,1)$ while the solution of class $(2,2)$ is a weak solution. 


\section{Example 4.2}

Consider the following FBVP

$\widetilde{y^{\prime \prime}}(t)=-5 \widetilde{y}(t)-4 \tilde{y}(t), \tilde{y}(0)=(0.8,1,1.4), \tilde{y}(1)=(2.6,3,3.1)$.

\section{Class $(1,1)$}

The final solution:

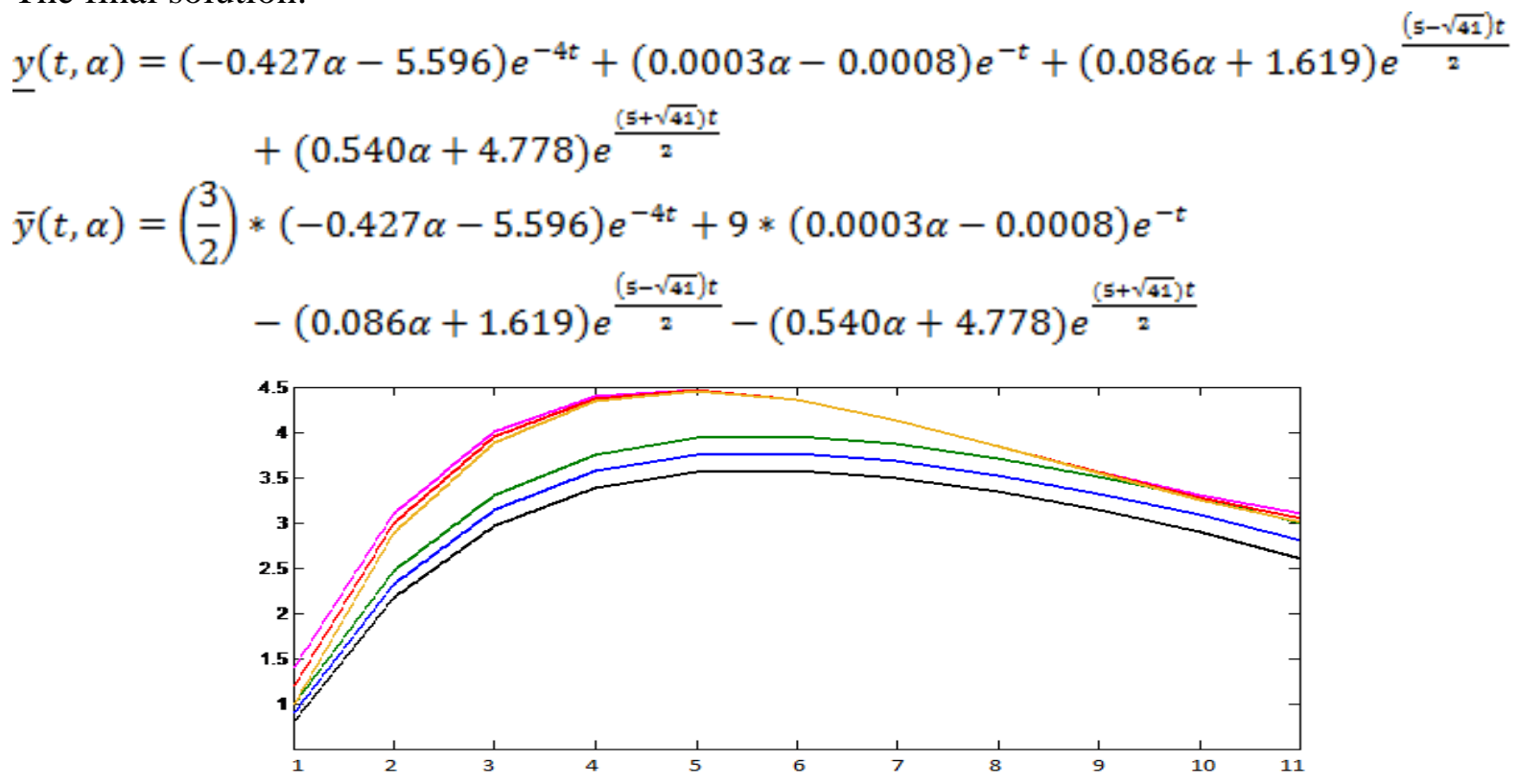

Fig. 7 the lower and upper solution class(1, 1) Example 2

We can easily notice the intersections and overlapping which starts at $\mathrm{t}=0.5$ till $\mathrm{t}=1$ and this indicates the weakness in the solution.

\section{Class $(1,2)$}

$y(t, \alpha)=(1.134 \alpha+7.396) e^{-t}+(-0.934 \alpha-6.596) e^{-4 t}$
$\bar{y}(t, \alpha)=(8.794-0.265 \alpha) e^{-t}+(-0.134 \alpha-7.395) e^{-4 t}$

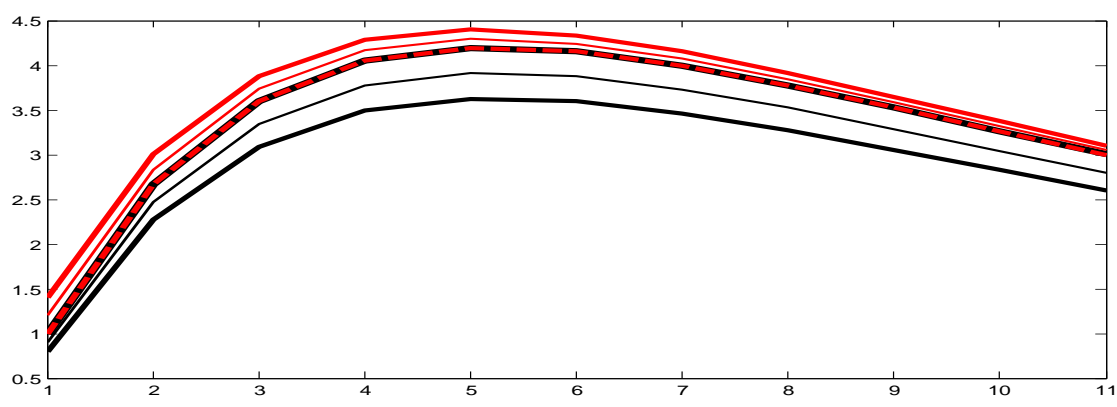

Fig. 8 the lower and upper solution class $(1,2)$ Example 2

The red waves represent $\overline{\boldsymbol{y}}(\boldsymbol{t}, \boldsymbol{\alpha})$ and the black waves represent $\underline{\boldsymbol{y}}(\boldsymbol{t}, \boldsymbol{\alpha})$ also we can see at $\alpha=1 \bar{y}(t, 1)=\underline{y}(t, 1)$ the crisp solutionbut only for the triangular fuzzy conditions. 
Class $(2,1)$

$$
\begin{aligned}
& \underline{y}(t, \alpha)=(0.0108-0.0108 \alpha) e^{4 t}+(0.434 \alpha+8.095) e^{-t}+(-0.534 \alpha-6.995) e^{-4 t} \\
& +(0.3109 \alpha-0.3109) e^{t} \\
& \bar{y}(t, \alpha)=-(0.0108-0.0108 \alpha) e^{4 t}+(0.434 \alpha+8.095) e^{-t}+(-0.534 \alpha-6.995) e^{-4 t} \\
& -(0.3109 \alpha-0.3109) e^{t}
\end{aligned}
$$

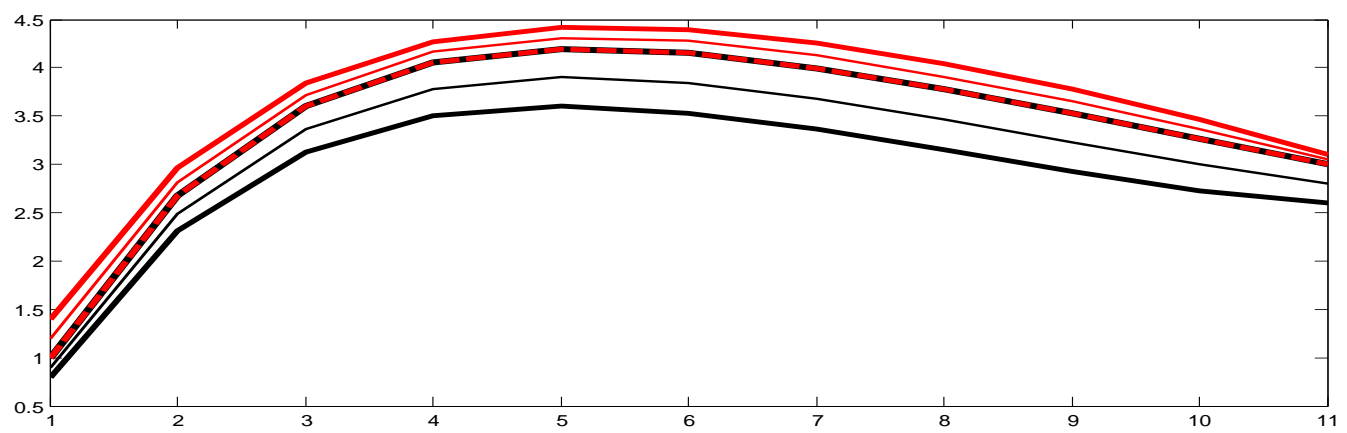

Fig. 9 the lower and upper solution $\operatorname{class}(2,1)$ example 2

The red waves represent $\overline{\boldsymbol{y}}(\boldsymbol{t}, \boldsymbol{\alpha})$ and the black waves represent $\underline{\boldsymbol{y}}(\boldsymbol{t}, \boldsymbol{\alpha})$ also we can see at $\alpha=1 \bar{y}(t, 1)=\underline{y}(t, 1)$ the crisp solutionbut only for the triangular fuzzy conditions.

\section{Class (2, 2)}

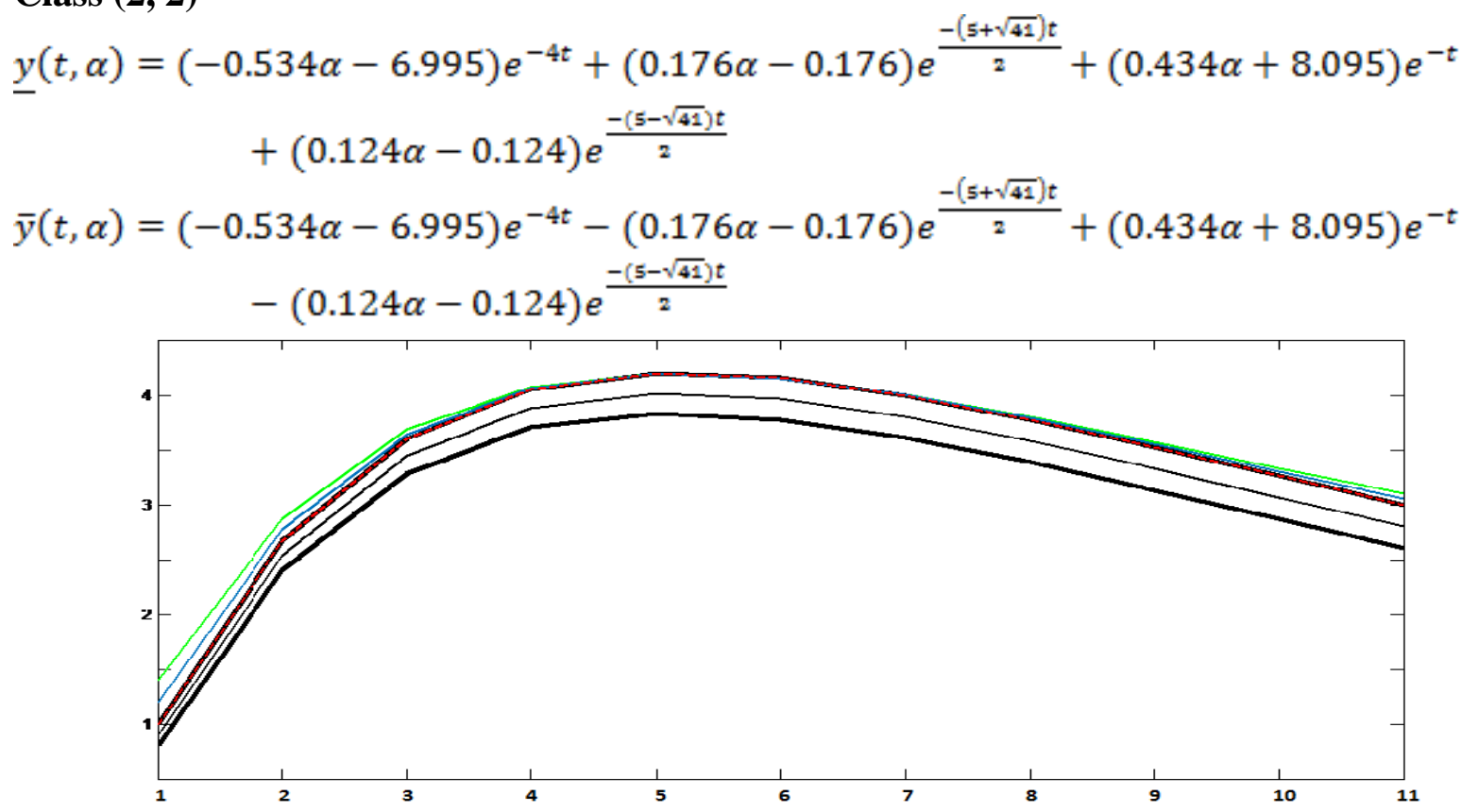

Fig. 10 the lower and upper solution class $(2,2)$ example 2

We can see some overlapping but it may be equal at point or so near wavebut, we can notice that waves are not intersected or by another words the lower bands don't 
exceed the upper bands but we can also see that the narrow region starts from $\mathrm{t}=$ 0.5 and 0.6 .

Table 3: The lower and upper solutions of Example 2 for $t=0.5$

\begin{tabular}{|l|l|l|l|l|l|l|l|l|}
\hline & \multicolumn{2}{l}{ Class $(1,1)$} & \multicolumn{2}{l|}{ Class $(1,2)$} & \multicolumn{2}{l|}{ Class $(2,1)$} & \multicolumn{2}{l|}{ Class $(2,2)$} \\
\hline$\alpha$ & $\underline{y}(0.5, \alpha)$ & $\bar{y}(0.5, \alpha)$ & $\underline{y}(0.5, \alpha)$ & $\bar{y}(0.5, \alpha)$ & $\underline{y}(0.5, \alpha)$ & $\bar{y}(0.5, \alpha)$ & $\underline{y}(0.5, \alpha)$ & $\bar{y}(0.5, \alpha)$ \\
\hline 0 & 3.5739 & 4.3528 & 3.5932 & 4.3336 & 3.5313 & 4.3954 & 3.7134 & 4.2134 \\
\hline 0.2 & 3.6502 & 4.3530 & 3.7055 & 4.2977 & 3.6560 & 4.3472 & 3.8016 & 4.2016 \\
\hline 0.4 & 3.7265 & 4.3531 & 3.8177 & 4.2619 & 3.7806 & 4.2991 & 3.8898 & 4.1898 \\
\hline $\begin{array}{l}0 . \\
6\end{array}$ & 3.8028 & 4.3533 & 3.9300 & 4.2261 & 3.9052 & 4.2509 & 3.9781 & 4.1781 \\
\hline 0.8 & 3.8791 & 4.3534 & 4.0422 & 4.1903 & 4.0299 & 4.2027 & 4.0663 & 4.1663 \\
\hline 1 & 3.9554 & 4.3536 & 4.1545 & 4.1545 & 4.1545 & 4.1545 & 4.1545 & 4.1545 \\
\hline
\end{tabular}

From table 3 we notice that in class $(2,2),(1,2)$ and $(2,1)$ the lower solutions at $t=0.5$ are increasing and the upper solutions are decreasing which means that these classes have a strong solution and we can find that the solutions at 1 are equal which strength the solution because of the triangle fuzzy number in conditions.

But class $(1,1)$ besides the overlapping shown in fig. 7 , we can notice the upper solutions are slightly increasing so we can easily conclude that it is weak solution.

\section{CONCLUSION:}

In this paper; the analytical solution of a second order differential equation with fuzzy conditions under generalized hukuhara differentiability. Here fuzzy numbers are taken as generalized trapezoidal fuzzy number.

The results are very useful in the field of differential equation theoretically and in the applications, it was effective in determining the strong and weak solutions.

In the future research we will solve the same problem but with adding a fuzzy nonhomogeneous term.

\section{REFERENCES:}

\begin{tabular}{|l|l|}
\hline$[1]$ & $\begin{array}{l}\text { A. Armand and Z. Gouyandeh, Solving two-point fuzzy boundary value problem using } \\
\text { variation iteration method, Communications on Advanced Computational Science with } \\
\text { Applications. 2013 (2013) 1-10. }\end{array}$ \\
\hline$[2]$ & $\begin{array}{l}\text { B. Bede and S. G. Gal, Generalizations of the differentiability of fuzzy-number-valued } \\
\text { functionswith applications to fuzzy differential equations, Fuzzy Sets and Systems 151 } \\
\text { (2005) 581-599. }\end{array}$ \\
\hline$[3]$ & $\begin{array}{l}\text { B. Bede, A note on two-point boundary value problems associated with non-linear fuzzy } \\
\text { differential equations, Fuzzy Sets and Systems 157 (2006) 986-989. }\end{array}$ \\
\hline$[4]$ & $\begin{array}{l}\text { B. Bede, I. J. Rudas and A. L. B Encsik, First order linear fuzzy differential equations } \\
\text { undergeneralized differentiability, Inform. Sci. 177 (2007) 1648-1662. }\end{array}$ \\
\hline$[5]$ & $\begin{array}{l}\text { B. Bede and L. Stefanini, Generalized differentiability of fuzzy-valued functions, Fuzzy } \\
\text { Setsand Systems 230 (2013) 119- 141. }\end{array}$ \\
\hline$[6]$ & $\begin{array}{l}\text { L. C. Barros, L. T. Gomes and P. A Tonelli, Fuzzy differential equations: An approach } \\
\text { viafuzzification of the derivative operator, Fuzzy Sets and Systems 230 (2013) 39-52. }\end{array}$ \\
\hline
\end{tabular}




\begin{tabular}{|c|c|}
\hline [7] & $\begin{array}{l}\text { Y. Chalco-Cano and H. Roman-Flores, on the new solution of fuzzy differential } \\
\text { equations, Chaos Solitons Fractals. } 38 \text { (2008) } 112-119 \text {. }\end{array}$ \\
\hline [8] & $\begin{array}{l}\text { Y. Chalco-Cano, H. Roman-Flores and M. A. Rojas-Medar, Fuzzy differential equations } \\
\text { withgeneralized derivative, in: Proceedings of the } 27 \text { th North American Fuzzy } \\
\text { Information Processing Society International Conference, IEEE. } 2008 \text {. }\end{array}$ \\
\hline [9] & $\begin{array}{l}\text { S. L. Chang and L. A. Zadeh, on fuzzy mapping and control, IEEE Transaction on } \\
\text { Systems Man Cybernetics } 2 \text { (1972) 30-34. }\end{array}$ \\
\hline [10] & $\begin{array}{l}\text { D. Dubois and H. Prade, towards fuzzy differential calculus: Part 3, Differentiation, } \\
\text { FuzzySets and Systems } 8 \text { (1982) 225-233. }\end{array}$ \\
\hline [11] & $\begin{array}{l}\text { P. Diamond and P. Kloeden, Metric Spaces of Fuzzy Sets, World Scientific, Singapore. } \\
1994 .\end{array}$ \\
\hline [12] & $\begin{array}{l}\text { R. Goetschel and W. Voxman, Elementary fuzzy calculus, Fuzzy Sets and Systems } 18 \\
\text { (1986)31-43. }\end{array}$ \\
\hline [13] & $\begin{array}{l}\text { N. Gasilov, S. E. Amrahov, A. G. Fatullayev and A. Khastan, A new approach to fuzzy } \\
\text { initialvalue problem } 18 \text { (2) (2014) 217-225. }\end{array}$ \\
\hline [14] & $\begin{array}{l}\text { N. Gasilov, S. E. Amrahov and A. G. Fatullayev, Solution of linear differential equation } \\
\text { withfuzzy boundary values, Fuzzy Sets and System } 257 \text { (2014) 169-183. }\end{array}$ \\
\hline [15] & $\begin{array}{l}\text { X. Guo, D. Shang and X. Lu, Fuzzy approximate solutions of second-order fuzzy linear } \\
\text { boundary value Problems, Boundary Value Problems } 2013 \text { (2013) } 212 \text {. }\end{array}$ \\
\hline [16] & $\begin{array}{l}\text { L. Jamshidi and L. Avazpour, Solution of the Fuzzy Boundary Value Differential } \\
\text { Equationsunder Generalized Differentiability by Shooting Method. } 2012 \text { (2012) 1-19. }\end{array}$ \\
\hline [17] & O. Kaleva, Fuzzy differential equations, Fuzzy Sets and Systems 24 (1987) 301-317. \\
\hline [18] & $\begin{array}{l}\text { A. Khastan and J. J. Nieto, A boundary value problem for second-order fuzzy } \\
\text { differentialequations, Nonlinear Anal } 72(2010) 3583-3593 \text {. }\end{array}$ \\
\hline [19] & $\begin{array}{l}\text { H. K. Liu, Comparison results of two-point fuzzy boundary value problems, World } \\
\text { Academyof Science, Engineering and Technology. } 512011 .\end{array}$ \\
\hline [20] & $\begin{array}{l}\text { M. L. Puri and D. A. Ralescu, Differentials of fuzzy functions, J. Math. Anal. Appl. } 91 \\
\text { (1983)552-558. }\end{array}$ \\
\hline [21] & $\begin{array}{l}\text { S. J. Ramazannia Tolouti and M. Barkhordari Ahmadi, Fuzzy Laplace transform on two } \\
\text { orderderivative and solving fuzzy two order differential equation, Int. J. Industrial } \\
\text { Mathematics.2(4) (2010) 279-293. }\end{array}$ \\
\hline [22] & $\begin{array}{l}\text { F. Rabiei, F. Ismail, A. Ahmadian and S. Salahshour, Numerical Solution of Second- } \\
\text { OrderFuzzy Differential Equation Using Improved Runge-Kutta Nystrom Method, } \\
\text { Mathematical Problems in Engineering. } 2013 \text { (2013) 1-10. }\end{array}$ \\
\hline [23] & $\begin{array}{l}\text { L. Stefanini and B. Bede, Generalized Hukuhara differentiability of interval-valued } \\
\text { functionsand interval differential equations, Nonlinear Analysis } 71 \text { (2009) 1311-1328. }\end{array}$ \\
\hline [24] & $\begin{array}{l}\text { L. Wang and S. Guo, Adomian method for second-order fuzzy differential Equation, } \\
\text { WorldAcademy of Science, Engineering and Technology. } 76 \text { (2011) }\end{array}$ \\
\hline [25] & $\begin{array}{l}\text { L. Zadeh, toward a generalized theory of uncertainty (GTU) an outline, Inform. Sci. } \\
172(2005) 1-40 \text {. }\end{array}$ \\
\hline [26] & $\begin{array}{l}\text { S.Mondal and T.Roy, Solution of second order linear differential equation in fuzzy } \\
\text { environment. }\end{array}$ \\
\hline
\end{tabular}

